\title{
GENERACIÓn, TRABAJO Y JUVENTUD. RELATOS DE VIDA DE JÓVENES MIXTECOS Y ZAPOTECOS EN EL CIRCUITO DE MIGRACIÓN RURAL HACIA LA FRONTERA NORTE
}

\author{
Susana Vargas-Evaristo
}

Resumen: En el presente artículo se plantea la siguiente pregunta: ¿qué ha significado en la biografía de jóvenes mixtecos y zapotecos crecer en un contexto de trabajo jornalero y movimientos migratorios en dos espacios de migración: el Valle de San Quintín, Ensenada, Baja California y Madera, California? Se recurre al enfoque generacional utilizando el concepto "situación de generación", entendido como aquel proceso que va más allá de la edad de los sujetos, tratando de enfatizar cómo ciertos acontecimientos sociales e históricos han trastocado la biografía de los individuos, como es el caso de los jóvenes que nacieron y crecieron en situación de migración. El trabajo concluye resaltando la importancia de abordar a los sujetos que se encontraron inmersos en procesos migratorios desde una perspectiva generacional y biográfica que estudie como procesos históricos sus historias de vida, articuladas a acontecimientos sociales.

Palabras clave: Migración, situación de generación, biografía, juventud.

Enviado a dictamen: 12 de abril de 2012

Aprobación: 25 de julio de 2012

Revisiones: 1

Mtra. Susana Vargas Evaristo, licenciada en Antropología Social por la Escuela Nacional de Antropología e Historia, maestra en Desarrollo Regional por El Colegio de la Frontera Norte, estudiante de doctorado en Antropología en el Instituto de Investigaciones Antropológicas y la Facultad de Filosofía y Letras de la UNAM. Temas de especialización: migración indígena interna e internacional. Correo electrónico: susana.vargas.e@gmail.com.
Abstract: This article raises the question: What has meaning in the biography of young Mixtec and Zapotec who grow in the agricultural labor and migration context, in two places: San Quintin Valley, B.C. and Madera, California? Generational approach was selected using the concept of "situación de generación" as the processes that go beyond the age of the subjects, trying to emphasize more on how certain social and historical events have disrupted the biography of individuals, as is the case of young people born in a situation of migration. This work conclude by the importance of addressing the subjects were involved in migration situation from a generational perspective and biographical study where stories are articulated to social events such as historical processes.

Keywords: Migration, generation status, biography, youth.

\section{"Situación de generación" como un proceso histórico}

Revista LiminaR. Estudios Sociales y Humanísticos, año 10, vol. X, núm. 2, julio-diciembre de 2012, San Cristóbal de Las Casas, Chiapas, México. ISSN: 1665-8027 163 
principal para hacerlo. Sin embargo, existen algunos aspectos que son útiles para analizar las generaciones en términos cronológicos. En el concepto acuñado por Mannheim sobre "situación de generación", vemos que el interés se centra no únicamente en el criterio de la edad, sino también en un tiempo y espacio históricos que son compartidos, así como "en las condiciones sociales y culturales en que viven los sujetos, pues son ellas las que en definitiva determinan los modos de experiencia y conciencia posibles" (Ghiardo, 2004: 24). Este compartir un tiempo y espacio históricos, una edad cercana o ser contemporáneos, produce en los individuos esquemas de ideas y actitudes que expresan la situación en conjunto de los sujetos. Algunos señalan que "el sólo hecho de haber nacido en un mismo momento de la historia supone ya un sentimiento de identidad y pertenencia a un colectivo" (Ghiardo, 2004: 31). Para estudiosos de la juventud como Feixa, la identidad de una generación es un fenómeno de relevancia, pues considera que "es el gran factor estructurador de las culturas juveniles" (Feixa, 1999: 89). En estas culturas se sintetiza, pues, un contexto histórico vivido por una generación, el cual no se rompe de una generación a otra, sino que más bien pueden coexistir conflictivamente y con sus respectivas continuidades.

Pensar las generaciones desde la perspectiva histórica nos permite entonces acceder al campo de la memoria y de la experiencia de las diferentes generaciones en un tiempo y espacio determinados. De esta manera, cada individuo toma contacto con el tipo de realidad en el que se encuentra inmerso, de tal suerte que las experiencias quedan impresas en su memoria y conciencia. En estas experiencias, la infancia cumple un papel fundamental al ser ésta la etapa de la vida en la que "se forman las capas primeras de la conciencia y permanecen como las bases desde las cuales interpretará en adelante su realidad" (Ghiardo, 2004: 37). Así vemos que vivir una misma "situación de generación" implica estar vinculados por la misma posición social, histórica y biográfica.
A este respecto, en su libro sobre narrativas de identidad de mixtecos migrantes hacia la frontera norte, Velasco (2005) ofrece una definición interesante sobre lo que llama el tiempo generacional, el cual incluye a los antepasados. La autora lo utiliza para distinguir el tiempo proyectado hacia el futuro a partir de las experiencias del pasado. En este sentido, el tiempo generacional va más allá de la temporalidad biográfica del individuo, es decir, si bien los vínculos consanguíneos y familiares son relevantes, existe una "idea generacional que se amplía a un ser colectivo con atributos étnicos y de clase: la gran parentela étnica empobrecida" (Velasco, 2005: 271).

Así, la concepción de generación debería ayudar a articular dos situaciones: 1) la que tiene que ver con el tiempo cronológico de un individuo, el cual atraviesa etapas de la vida tales como la infancia, la adolescencia y la juventud, y 2) sin embargo, este tiempo necesariamente está vinculado con un tiempo y espacio históricos. Por ello, a través de las narrativas de los sujetos podemos observar el pasado, el presente y de alguna manera el futuro, pero no únicamente de un sujeto en sí, sino de un grupo social que ha quedado envuelto o sumergido en dinámicas sociales determinadas. De esta manera, si bien se puede partir de relatos de vida individualizados, esto no necesariamente significa que pueda haber detrás una colectividad identificada por ciertos atributos: los de clase y etnicidad sólo son algunos de ellos.

\section{Situando al sujeto de estudio}

Situar a los sujetos en procesos específicos tales como la agricultura de exportación y los circuitos de migración interna e internacional nos parece adecuado en tanto permite preguntarnos iqué sucede en la biografía de un sujeto que ha nacido y crecido al ritmo de los movimientos migratorios de su familia en busca de trabajo para sobrevivir como grupo? Desde la perspectiva de Seyla Benhabib (2002), los sujetos 
son agentes capaces de producir cultura mediante sus propios sentimientos de pertenencia y narrativas de identidad. Así vemos que, dentro de la migración indígena a regiones agrícolas como proceso histórico, podemos construir un sujeto de estudio, tal como los jóvenes indígenas que nacieron y crecieron en contextos de migración insertos en la agricultura de exportación, y observar los efectos que esto tuvo en la constitución de su biografía. De esta manera, el objetivo del presente artículo es describir y analizar, a partir de información empírica, qué ha significado el trabajo jornalero agrícola en la biografía de jóvenes mixtecos y zapotecos que crecieron y nacieron en espacios de migración.

Para efectos de nuestro estudio nos concentramos sólo en dos puntos de atracción de migración indígena oaxaqueña pertenecientes a dicho circuito de migración agrícola: Madera-Fresno, California, y el Valle de San Quintín, Baja California. Se eligieron estas regiones porque ambas han sido consideradas como las de mayor atracción de mano de obra indígena oaxaqueña, además de presentar un importante proceso de asentamiento (National Agricultural Workers Survey, 1991-2008).

La información de la que se nutre el presente artículo proviene de relatos biográficos realizados con jóvenes de familias migrantes pertenecientes a los grupos étnicos zapoteco y mixteco. Las edades están organizadas en dos grupos: el primero de 17 a 25 años y el segundo de 26 a 35 años. El primer rango de edades corresponde a la generación de jóvenes que nació durante los años ochenta y el segundo a los que nacieron en los años noventa en los espacios de migración. Portes (1996) y Zhou (1997) han discutido ampliamente sobre el tema de las segundas generaciones. Utilizando sus criterios de definición, en el presente trabajo se considerará a los que nacieron en el lugar de origen como generación intermedia, mientras que al grupo que nació en el lugar de destino, lo consideraremos segunda generación.

\section{Circuito de migración rural hacia la frontera norte} (México-Estados Unidos)

Desde hace varias décadas se ha venido conformando un circuito de migración indígena que iniciaba en Veracruz, Morelos, Sonora, Sinaloa y Baja California. Familias enteras se fueron desplazando siguiendo la producción de hortalizas en el marco de una agricultura de exportación claramente insertada en una economía global, conectada a través de su frontera con la producción del noroeste estadounidense que comprende los estados de California, Oregón y Washington.

Por su parte, la región noroeste de México se fue conformando como un nodo de atracción de población indígena proveniente principalmente de la mixteca de Oaxaca y Guerrero. ${ }^{1}$ Como resultado de una producción anual continua, el Valle de San Quintín comienza a establecerse como un sitio de paso obligado e incluso de asentamiento para las familias migrantes.

Diversos estudios han documentado cómo a partir de los años ochenta ${ }^{2}$ se produjo un proceso de asentamiento intensivo en la región del Valle de San Quintín (Camargo, 201l; Coubès, Velasco y Zlolniski, 2009) como resultado de un aumento en la demanda anual de mano de obra en el mercado de trabajo agrícola (Velasco, 2002). En este mismo periodo, pero en el lado estadounidense, se otorgaba amnistía a los trabajadores mexicanos mediante la IRCA ${ }^{3}$, lo cual estimuló la incorporación de migrantes indígenas a los campos californianos. De esta manera, San Quintín se fue conformando como una región intermedia de migración que "comunica" la migración interna con la migración internacional. En este contexto ocurren dos situaciones históricas: por una parte, el proceso de asentamiento que fue desalentando la migración circular y, por otra, la reunificación familiar, particularmente en el caso de los que se encontraban en California.

Es entonces en este periodo, en las décadas de los ochenta y los noventa, donde situamos una 
generación de infantes migrantes indígenas que crecieron (y nacieron) en el contexto de un circuito de migración rural para incorporarse al mercado de trabajo agrícola inserto en una economía global. Una característica común a esta generación es su constante inserción en los campos como trabajadores, muchos de ellos empleándose directamente como jornaleros y otros como ayudantes de sus padres y hermanos o participando en actividades domésticas en apoyo a la sustentabilidad de sus hogares mientras sus padres salían a trabajar en los campos, tanto de California como de Baja California.

\section{El Valle de San Quintín. Relatos de salida de la generación intermedia y de la segunda generación}

El grupo de informantes de la que llamamos generación intermedia está conformado por los jóvenes adultos de entre 26 y 35 años que llegaron al Valle durante su infancia en los años ochenta. Son distintos los aspectos que caracterizan a este primer grupo, uno de estos es el proceso de conformación de su propia familia realizado durante los recorridos itinerantes de sus padres en la región noroeste del país. Estos jóvenes desde su infancia enfrentaron procesos de movilidad constante tanto a nivel regional como local. Al tiempo que la familia se desplazaba de un lugar a otro en busca de contratarse en los campos agrícolas, iban naciendo los miembros de la familia, de ahí que algunos de los hermanos y hermanas nacieran en el pueblo de origen, otros en Sinaloa y otros más en San Quintín, es decir, el proceso de conformación de la familia se dio dentro de estos itinerarios migratorios. Como consecuencia de la insuficiente entrada económica, el crecimiento de la familia y la poca estabilidad residencial, los jóvenes durante su infancia se vieron obligados a trabajar en los campos agrícolas, ya sea haciendo tareas específicas como el corte de tomate, o apoyando a sus padres y hermanos mayores:
Susana: Mariana, no sé si tú tuviste experiencia de migración, si anduviste digamos en varias regiones, en diferentes campos con tu familia.

Mariana: Sí, nosotros, bueno, mi familia, nos venimos de allá cuando yo tenía tres años, salimos de Oaxaca, llegamos a Mexicali a un ejido que se llama Piedras Negras, ahí llegamos porque mis papás trabajaban en el algodón y en la uva, ahí estuvimos y ahí cursamos, bueno yo cursé lo que era primer año de primaria ahí en Mexicali. Ahí estuvimos, pues ya nos venimos de ahí cuando yo tenía 6,7 años, y llegamos a trabajar a una compañía que se llamaba de los Canelos ABC [En el Valle de San Quintín] (Mariana [pseudónimo] 17 de agosto de 2010, San Quintín, B.C.).

El Valle de San Quintín representó para muchas familias oaxaqueñas una buena posibilidad de establecerse en tanto era el lugar donde encontraban mejores condiciones climáticas para trabajar. Existen algunos relatos que muestran las condiciones de Sinaloa, donde el calor los asediaba y hacía más duro el trabajo en el campo. Otro aspecto importante para las familias era la posibilidad de incorporar a todos sus miembros al trabajo en el campo. En San Quintín, durante varias décadas existieron empresas agrícolas donde se formaban cuadrillas de niños y niñas jornaleros. Según los relatos biográficos, fueron los jóvenes que llegaron durante su infancia al Valle los más susceptibles de incorporarse a la vida laboral en los campos.

A pesar de que el aporte económico que los niños daban al grupo familiar se veía como periférico, ciertamente en los relatos de vida es notable observar que muchas veces, con este ingreso, sostenían sus estudios eincluso los de sus hermanos menores. Cuando trabajaban y recibían un pago, éste lo entregaban íntegro a su mamá o papá, según correspondiera. Muchas veces el dinero familiar fue utilizado para la compra de un terreno, debido a que durante los años ochenta se dio un proceso de asentamiento intensivo en el Valle, lo que les permitía a las familias itinerantes pasar de residir 
en campamentos ${ }^{4}$ a vivir en colonias donde tenían la posibilidad de construir su propia vivienda.

Así vemos que un segundo aspecto importante es el tránsito de vivienda entre los campamentos y las colonias. Este proceso fue vivido por los jóvenes migrantes de la generación intermedia y no por los que ya nacieron en el Valle. Pero iqué pudo significar en sus vidas haber vivido en campamentos y posteriormente en colonias? Un hecho sustancial fue la inserción escolar y la desincorporación del trabajo en los campos. En los campamentos, la familia completa se veía obligada a trabajar diariamente, mientras que en las colonias, la elección de dejar a los hijos en la escuela durante la jornada laboral de los padres comenzaba a ser una opción. En las colonias existía un acceso más seguro y constante a las escuelas que en los campamentos, bien porque no había escuelas, o simplemente porque, por la misma movilidad de la familia, los niños y las niñas se veían obligados a cortar sus ciclos escolares. Además, cabe señalar que las condiciones de vida en los campamentos muchas veces eran deplorables para los niños y las niñas: muchos de ellos estaban expuestos a situaciones de violencia, no sólo a manos de sus propios familiares, sino también de las demás familias con las que convivían bajo el mismo techo.

A partir del cambio residencial, muchas familias comenzaron a construir sus casas con materiales de desecho de los campos, como cartones, palos, plásticos o mallas. Levantaban un cuarto para cubrirse de la intemperie y con el tiempo continuaban construyendo con materiales más duraderos. Si bien la desincorporación de los menores del trabajo jornalero no fue total, ciertamente comenzó a aparecer la opción de trabajar y estudiar durante los fines de semana o en vacaciones. La asistencia a la escuela entonces comenzó a ser una posibilidad para los jóvenes durante su infancia. Su inserción escolar muchas veces se tornaba problemática ya que representaba un conflicto generacional e incluso de género. Los padres consideraban que el objetivo principal de haber migrado a San Quintín era trabajar y no asistir a la escuela. Finalmente, por estudiar no había una remuneración económica y prevalecía la idea de que "esto no es para nosotros”. Por otra parte, bajo la percepción de que las mujeres tienen que casarse, los padres consideraban innecesario que sus hijas estudiaran, como también así lo consideraban para sus hijos varones ya que éstos lo que requerían era trabajar para mantener a una familia:
...empecé a escuchar que había una prepa, que habían abierto una extensión aquí por el [ejido] Raúl Sánchez Díaz, donde está la [Escuela] Normal, que ahí habían abierto la extensión de una prepa, pues igual hablé con mi familia y les dije [a mis papás] que quería estudiar la prepa, pero que ahí había que pagar una inscripción y pues yo quería dinero y que no les iba a dar mi cheque. Pues mi mamá dijo que estaba bien, pero pues mi papá no, dijo que la escuela no era para nosotros porque él no sabe leer ni escribir, entonces dijo que él no sabía ni leer ni escribir y comía, entonces me dijo, para qué quieres ir, eso no es para ti, no se hizo para nosotros, mírate, mírate bien... (Mariana [pseudónimo] 17 de agosto de 2010, San Quintín, B.C.).

La educación aparece en los relatos de los jóvenes como un factor que nos muestra una suerte de condición étnica y de clase por la que la población indígena históricamente ha atravesado, esto es, la idea de que los indígenas sólo son funcionales para trabajar en el campo, en condiciones hostiles, pero no para tener acceso a una educación y procurarse un estilo de vida diferente. Sin embargo, entre el trabajo, los conflictos familiares y económicos, las condiciones de pobreza y la hostilidad, los jóvenes que entrevistamos lograron terminar al menos el nivel medio superior, otros lograron acceder a la universidad e incluso actualmente estudian posgrados.

¿Cuál es el escenario que se les presentó a los jóvenes de segunda generación en el Valle de San Quintín 
durante su infancia? Existen algunas diferencias con respecto a los que llegaron siendo niños. En primera instancia, este grupo de jóvenes no experimentó la vida itinerante en busca de trabajo tanto en otras regiones como de manera local. Regularmente son los miembros más pequeños de la familia y sus edades oscilan entre los 17 y los 25 años — pertenecen a la generación de los noventa- Esto supone que tuvieron acceso a mejores condiciones de vida y la posibilidad de incorporarse a la escuela desde su infancia sin cortar sus ciclos escolares. Regularmente se puede observar que este segmento de los jóvenes de segunda generación ha tenido el apoyo económico tanto de sus padres como de sus hermanos mayores, e incluso han tenido la posibilidad de viajar a la ciudad de Ensenad $a^{5}$ para realizar estudios universitarios.

Susana: ¿Cómo le hacías para mantener tus estudios en Ensenada?

Virginia: ¿Allá? pues mis papás me mandaban de aquí [de San Quintín], como allá yo no trabajo pues, porque sí al principio cuando me fui mis papás me dijeron que me apoyaban. Bueno, no me dijeron, pero pues yo pensé que como ellos tienen la tienda a veces es mucho gasto, pues, porque los tres [hermanos] íbamos a estar estudiando y ya dije, no pues yo también tengo que aportar algo, y ya decidí buscar trabajo, pero no encontré, sólo encontré en tiendas o así, tiendas así de ropa o zapaterías, te pedían con horario flexible y pues yo no cuento con eso... (Virginia [pseudónimo] 15 de agosto de 2010, San Quintín, B.C.).

Por otra parte, el haber crecido en colonias les ha posibilitado tener un tipo de arraigo distinto al del Valle en comparación con los jóvenes de la generación intermedia. Su forma de identificarse es como sanquintinenses con "orígenes" o "raíces" en Oaxaca, mientras que, en el caso del primer grupo, se identifican como originarios de Oaxaca y han ido desarrollando un sentido de pertenencia a San Quintín.
Sin embargo, el trabajo jornalero y el proceso de migración que vivió su familia ha trastocado sus vidas. Eventualmente se incorporan al trabajo en los campos como una forma de apoyo económico familiar, para juntar dinero, comprar uniformes, enseres escolares o ropa y accesorios de su gusto. Así vemos que, en el primer grupo de jóvenes, su participación en la economía familiar durante la infancia resultaba de primera necesidad, mientras en el segundo grupo se considera eventual y muy enfocada a sus propias necesidades.

En el caso de la generación intermedia es de notarse que no necesariamente existe un conflicto con sus padres. Puede decirse que la generación anterior colocó las bases para que el tema de la escolaridad se observara como parte de las actividades de los niños y niñas. Además, cabe decir que, en las últimas décadas, en el Valle de San Quintín las instituciones educativas han crecido de manera importante, lo que nos habla de una presencia mayor de jóvenes que en décadas anteriores.

\section{Madera-Fresno, California. Relatos de salida de la generación intermedia y de segunda generación}

En el caso de la migración internacional, la situación se vuelve más compleja: no solamente existe la segunda generación y la generación intermedia de jóvenes, sino los estatus migratorios de residencia, ciudadanía y sin documentos. Trataré de ser breve en las descripciones con el fin de mostrar las características más importantes en cuanto al momento de la infancia para ambas generaciones de acuerdo con su estatus migratorio.

En la generación intermedia están concentrados los jóvenes que cuentan con residencia y aquellos que aún no tienen documentos para vivir legalmente en el país. En el caso de los residentes, éstos llegaron durante su infancia a Estados Unidos. La mayoría de ellos tuvo la experiencia de formar parte del circuito migratorio agrícola en México hasta que posteriormente sus padres regresaban por ellos al pueblo para llevárselos a vivir a 
Fresno o Madera, California, donde está asentada una gran comunidad de indígenas oaxaqueños provenientes de diversos pueblos mixtecos y zapotecos. El ritual de migración consistía en que primero iba el padre solo a trabajar a los campos agrícolas de Fresno o Madera. Después de un largo periodo de idas y vueltas entre el pueblo y Estados Unidos, decidía llevarse a la familia completa a vivir con él, lo que significaba que los niños pasaran como indocumentados a dicho país. Cuando los padres contaban con residencia, obtenida durante la Amnistía de 1986, cruzaban sin problema la garita con sus hijos. Muchas veces se le indicaba al niño que se durmiera para que el oficial fronterizo no le hiciera ningún tipo de preguntas. Las familias que no se beneficiaron de la amnistía migraron de manera indocumentada cruzando por lo que llaman "el cerro", es decir, por la garita de Tijuana o por Tecate. En el caso de Mónica, que tenía seis años cuando sus padres decidieron llevarla a vivir a Fresno, su cruce fue por la garita de Tijuana-San Diego:

Susana: Mónica, me podrías platicar el proceso de migración que tú tuviste de niña, o sea de tu pueblo de origen hacia Estados Unidos, ¿cómo fue?

Mónica: Bueno, todo empezó, mi papá se vino primero, cuando se vino llegó a Oregón.

Susana: En qué año, ite acuerdas?

Mónica: Fue más como en el 85, unos meses después de que nací él se vino, él llegó primero a Oregón, y ya estuvo ahí creo como unos cinco años, y después él se vino para Fresno, California, y mi mamá se vino tal vez como a los dos años cuando yo tenía dos años de edad porque mi papá la mandó a traer. Y bueno, pues ella se vino con mi papá y ya básicamente yo me crié con mi abuelita, mi tía y mi tío, y no fue sino hasta los seis años de edad que mi mamá y mi papá iban con la intención de que yo me fuera con ellos y yo no sabía nada, y pues mi abuelita tampoco y así es como yo llegué hasta acá. (Mónica [pseudónimo] 3 de septiembre de 2010, Fresno, Ca.).
En el caso de los jóvenes cuyos padres habían obtenido la residencia en los ochenta, fue relativamente sencillo conseguir la suya y, posteriormente, realizar el trámite para obtener la ciudadanía por naturalización. La situación no es igual para los jóvenes que migraron de manera indocumentada junto con sus familias. Para ellos, la esperanza de obtener documentos migratorios cada vez se hace más lejana.

Las condiciones de vida en Estados Unidos para los residentes y para los indocumentados son diferentes. En el caso de los primeros, existen mayores posibilidades de estudiar y conseguir apoyos económicos del gobierno para continuar sus estudios universitarios. En el caso de los indocumentados, además del estrés y la incertidumbre que implica no contar con documentos, sus opciones de obtener financiamiento para sus estudios son reducidas. Además, su campo laboral se limita a su inserción en los campos agrícolas o en trabajos donde antes tienen que acordar con el patrón que los acepten con su estatus migratorio. Sara, zapoteca de 23 años, nos narra sus percepciones sobre su llegada a Estados Unidos y las diferencias entre los jóvenes indocumentados y los residentes:

...yo creo que la desventaja mayor es de los que no tienen documentos. Nosotros venimos aquí no porque queríamos venir, no porque yo quería venir estoy aquí, ¿no? sino porque fue una necesidad, fue ahora sí como que mis padres se sacrificaron más de 16 años, más de 17, 18 años estar separados, de estar como pareja tres meses, cuatro meses al año, ino?, y estar en una relación más que nada lejana... Creo que la mayoría de la gente, pues los jóvenes, no sabemos a qué venimos y cuando los jóvenes llegan sin documentos no les dan ni la oportunidad, todos estamos aquí por una razón y si ellos quieren ir a la escuela el hecho de que tienen que pagar por su colegiatura que son más de 200 mil dólares aquí en la universidad es como eso de que ayudas a tu familia o tratas de sobrevivir tú para pagar la escuela iy qué pasa después cuando termines? 
¿Puedes seguir estudiando? ¿O puedes trabajar en la carrera que estudiaste? porque no se puede, porque tienes que tener seguro bueno y porque te requieren tus papeles, entonces existe una mayor desventaja en ellos que los que tenemos residencia ino? (Sara [pseudónimo] 7 de septiembre de 2010, Madera, Ca.).

Por haber nacido en Estados Unidos, el grupo conformado por la segunda generación tiene muy pocos recuerdos de la historia de migración de su familia. Lo poco que pueden reconstruir en su memoria sobre tal proceso lo hacen a partir de pláticas que escuchan de sus hermanos o padres. Estos jóvenes forman parte de familias mixtas donde algunos de sus hermanos han nacido en el pueblo de origen, otros en la región noroeste de México y, finalmente ellos, en Estados Unidos. En el caso de estos jóvenes, su infancia transcurrió en la escuela. El manejo del idioma inglés, en comparación con los de la generación intermedia, era evidentemente mejor debido a que desde pequeños estuvieron expuestos al idioma, lo que hizo más sencilla su incorporación a la escuela. En el caso de la generación intermedia, este proceso fue complicado pues muchos pasaron de hablar su lengua materna, como el zapoteco, a comunicarse en inglés, siendo el español un idioma aprendido ya en su infancia tardía.

En cuanto a su inserción laboral, existen casos en los que los jóvenes relatan su interacción en los campos agrícolas, sin embargo, su contexto familiar y, desde luego, el entorno social, influían para que se priorizara la asistencia escolar antes que entrar a trabajar al campo. Como sucede en este tipo de migración rural e indígena, si bien algunos miembros de la familia no vivieron con intensidad su incorporación a los campos agrícolas desde su infancia, es cierto también que, como sus padres o hermanos debían trabajar, muchos tenían que dedicarse al cuidado de sus hermanos menores. Esto quiere decir que no necesariamente eran jornaleros, pero sí contribuían en actividades domésticas para que sus padres salieran a piscar a los campos, ya fuera en California (Fresno, Madera) o en Oregón.

Es en esta generación en la que las distancias y los conflictos generacionales son más pronunciados. Existe una suerte de dilema entre los jóvenes y las "costumbres" de sus padres en cuanto a diversos temas. Los jóvenes nacidos en el lugar de origen, de alguna manera cuentan con un background que les permite transitar entre la cultura de sus padres y la cultura a la que ahora están expuestos. Esto, en buena medida, se puede explicar por la cercanía y la interacción constante con el lugar de origen y con otros actores como los abuelos o los padres. La lengua en esta generación formó parte fundamental de este background, ya que los jóvenes de generación intermedia tienen nociones de la misma. Esto de alguna manera redunda en un vínculo directo de identidad.

En el caso de los que nacieron en Fresno o Madera, es complicada la situación debido a que están más vinculados a la cultura norteamericana. No podemos hablar de rupturas culturales, sino más bien de negociaciones constantes con la familia en términos de lo que los padres consideran como el rol correcto de un hombre y una mujer, entre otros temas. Considero que las negociaciones, y no los rompimientos, se producen porque esta generación de jóvenes trata de individualizarse, aunque el peso de la familia aún sigue siendo importante para ellos. Existe un "ir y venir" en términos de una necesidad de responder a un modelo de vida persistente en la sociedad receptora, no obstante, está presente el significado, la importancia y el peso que tienen las relaciones de parentesco dentro de las familias indígenas.

\section{Los dilemas del "ser joven" en el contexto de migración y el trabajo jornalero}

Por razones de espacio, sólo presentaré cuatro casos de jóvenes (mujeres y hombres) de origen mixteco y zapoteco con el objetivo de ilustrar cómo el trabajo y los procesos de migración en los que se ven involucrados 
afectan las formas en las que se sitúan en cada etapa de su vida, en este caso hablamos del período llamado juventud.

Caso Alejandro: Alejandro es un joven mixteco de 29 años que se dedica a estudiar el posgrado en contaduría en laUniversidad Autónoma de Baja California de San Quintín. A los nueve años llegó al Valle de San Quintín y entró a la escuela, pero posteriormente tuvo que incorporarse al trabajo jornalero. Alejandro narra cómo vivió su experiencia de trabajo en el campo:

\begin{abstract}
Alejandro: Mmm, pues cuando eres niño y vienes de una zona donde tienes que trabajar a fuerza pues no lo resientes.

Susana: O sea tu idea era trabajar.

Alejandro: Sí, porque así te lo enseñan en tu casa, mira, poco a poquito te van enseñando a trabajar y te van diciendo que tienes que trabajar, entonces ya traes la mentalidad y cuando ya empiezas a trabajar pues no lo resientes tanto, [...] sí resientes que es de sol a sol, este, a lo mejor te dan algún trabajo pesado, pero en ese entonces cuando yo empecé a trabajar, aquí en la zona había ranchos que tenían como grupos de niños haciendo trabajos no muy pesados. (Alejandro [pseudónimo] 16 de agosto de 2010, San Quintín, B.C.).
\end{abstract}

Cuando Alejandro llega a la adolescencia comienza a dar prioridad a sus estudios y se desinteresa por continuar apoyando a sus padres en el trabajo en el campo, por lo que comienza a haber un conflicto entre sus padres y él pues consideraban que un hombre que estudiaba no era productivo:

...vienes de una familia, o vienes de un lugar donde te dicen "itienes que producir!", o "itienes que ser productivo!", y empiezas a estudiar y las personas que son mayores y que tuvieron muchos años en el rancho, pues de alguna manera te dicen, "no estás produciendo", estás perdiendo tu tiempo, y tú te das cuenta de que pues no tienes ingresos, puros gastos, y te preguntas, itendrán razón?, o sea ivale la pena estudiar? Entonces sí llegas así como que a hacer un balance ¿no? A ver si es más bien la escuela o salirte de la escuela y a producir. (Alejandro [pseudónimo] 16 de agosto de 2010, San Quintín, B.C.).

Para la familia de Alejandro, estudiar significaba perder el tiempo y, de alguna manera, desatender las obligaciones que le corresponden a un joven en edad de matrimonio. Ser productivo y trabajar son dos aspectos de la vida masculina importantes de atender durante lo que podemos llamar la adolescencia y que cruzan la experiencia no sólo juvenil, sino también de género.

Caso José: José proviene de una familia zapoteca que reside en Madera, California. Tiene 22 años. Llegó a Estados Unidos cuando tenía diez y hasta el momento no cuenta con documentos migratorios. Estudia en el Colegio Comunitario de Madera y participa en varias organizaciones con diferentes perspectivas (comunitarias, de jóvenes, migrantes). Para mantener sus estudios, José trabaja por temporadas en los campos de Madera junto con su familia. Sobre la etapa de la juventud expresa lo siguiente:

Susana: ¿Qué significa para ti ser joven? ¿Tú vives esa parte de tu vida?

José: No tengo una respuesta concreta, deja ver, si tal vez con la explicación que te doy tú decides si vivo la juventud o no. Mira, desde muy niño mis papás me pusieron a trabajar, no me pusieron a trabajar por ser malos papás, sino por la situación en la que nos encontrábamos en ese entonces. En los noventa mis hermanos emigran para acá y me convierto yo casi en el hombre de la casa porque me quedé yo y mi mamá, y teníamos animales, tenemos chivos, o sea ya sea mi mamá cuidándolos o yo [...]. (José [pseudónimo] 2 de septiembre de 2010, Madera, Ca.). 
En el caso de José, además de provenir de un contexto cultural donde el trabajo se convierte en una de las principales funciones de los hombres, al migrar su papá y sus hermanos a Estados Unidos queda a cargo de su casa. Aunado a ello, cuando su familia decide llevárselo a Madera se ve obligado a trabajar arduamente para juntar dinero y poder pagar las cuotas del Colegio Comunitario, ya que tiene como propósito continuar estudiando mientras pueda estar en dicho país:

Así es como se le mete la idea a uno de niño ino? y ya así pasé mi preparatoria... Mucha gente me ha llamado la atención y me dice, "no, José, lo que estás haciendo te estás matando bastante, sí estás en la escuela, pero también déjate un tiempo para ti", pero yo creo que, dado a las barreras en las que me encuentro yo, pues yo creo que estoy en una situación muy difícil ¿no? Uno, yo no tengo quien paga por mi escuela, si yo quiero estudiar yo tengo que ganármelo de mi sudor iverdad? Otro, este, que no tengo los documentos, mi mentalidad dice... si yo tengo la mentalidad de llegar a ser como John Smith, bueno, tengo que reconocer que yo no soy nacido aquí, tengo que reconocer que es la realidad, mi piel es morena, o sea si yo quiero llegar a ser lo mismo que John Smith aunque vamos en la misma escuela, yo tengo que trabajar lo doble, entre otras cosas ino? Por ejemplo, John Smith, su lengua natal es el inglés, so, lo domina perfecto, en cambio yo no, yo tengo que recordar que hablo zapoteco y si yo sueño ser como John Smith, que es como lograr el sueño americano ino?, yo tengo que trabajar como burro para llegar ahí, sí, yo tengo, y esa es la mentalidad que yo tengo, quiero llegar a ser alguien.

... entonces, dadas las circunstancias, pues muy poco tiempo me dedico a la vida de juventud, pero yo creo que lo que yo hago no es mal gastado, yo creo que todo lo que hago es por mi beneficio personal y más que nada por el beneficio de la gente [por la] que nosotros abogamos, verdad, y me siento bien, yo hasta ahorita no me siento como "ay, me arrepiento" porque yo todo lo que hago lo hago con mucha pasión. Esto es lo que me gusta, es por eso que elijo una carrera de Ciencia Política, o sea, esto es mío, para mí es, pero si lo ponemos a analizar yo creo que vivo más la juventud, que un joven que piensa que la está viviendo. (José [pseudónimo] 2 de septiembre de 2010, Madera, Ca.).

Es interesante observar en el relato de José cómo, en el caso de los jóvenes que se encuentran en el contexto de migración internacional, además de por el trabajo y la familia sus relatos de vida se encuentran atravesados por otras categorías que afectan su vida juvenil. Por ejemplo, el hecho de ser indocumentado coloca a José en una situación de desventaja con respecto a aquellos jóvenes que tienen documentos como la ciudadanía o la residencia. Por otra parte, su origen étnico ha tenido impactos a lo largo de su vida, no sólo en Estados Unidos, en donde se evidencia más su desventaja étnica, sino igualmente en México, al igual que muchos otros jóvenes migrantes itinerantes en las regiones de agricultura de exportación del noroeste de México. Finalmente, lo que José resuelve en su relato es pensar que probablemente él, con todas las actividades que realiza (estudiar, trabajar y movilizar a su pueblo), vive de manera intensa su juventud, a diferencia de aquellos jóvenes que creen estar viviéndola. Esto nos permite entender cómo, de acuerdo a factores como el origen étnico, el estatus migratorio y las circunstancias que se les presentan, los sujetos interpretan el significado que le dan a la juventud.

Caso Sara: Sara es una joven de origen zapoteco. Tiene 23 años y llegó a California a los 14 años en calidad de residente debido a que su papá obtuvo amnistía durante los ochenta. Es estudiante de posgrado en la Universidad de Fresno State, además de activista social. En su relato de vida, Sara reflexiona sobre cómo su etapa juventud se vio trastocada por diversas circunstancias:

Revista LiminaR. Estudios Sociales y Humanísticos, año 10, vol. X, núm. 2, julio-diciembre de 2012, San Cristóbal de Las Casas, Chiapas, México. ISSN: 1665-8027 
Pues te voy a ser sincera, yo sentí que no tuve juventud ni allá como acá, mi vida fue diferente... Cuando estaba en Oaxaca era la de en medio, mi hermana estaba más chiquita, mi mamá se la pasaba yendo al campo o trabajando la tierra porque mi papá no estaba, entonces yo me quedaba al frente de la casa, tenía que hacer el trabajo porque cuidaba a mi hermana chiquita porque era la de en medio. Mi hermano era más grande y él no quería hacer las cosas, entonces, cuando yo estaba allá no sentí que tuviera tanta - a pesar de que yo estaba en mis 16 años ino? - no sentí como que tuve esa libertad de decir, oh, soy joven, irme a la disco a las movies inada! o irme al cine o algo así, iba a la escuela, iba al CBTIS y del CBTIS a mi casa... (Sara [pseudónimo] 7 de septiembre de 2010, Madera, Ca.).

La migración ha sido un factor fundamental en las concepciones que los jóvenes indígenas tienen de su mundo inmediato. Es común encontrar relatos de jóvenes mujeres en los que la ayuda en el cuidado de sus hermanos menores se torna una necesidad de la organización familiar, ya sea porque sus padres salen al campo a trabajar o porque, como es el caso de Sara, la familia está dividida entre Estados Unidos y el pueblo de origen. Esta situación nos permite ver que, aunque muchos de los jóvenes que ahora entrevistamos no se incorporaron directamente a las actividades del campo, como los padres y las madres debían cubrir largas jornadas de trabajo, las hijas mayores dedicaban su tiempo al cuidado de sus hermanos menores, de tal suerte que su participación formaba parte fundamental de la organización económica del grupo familiar.

Caso Catalina: Catalina nació en Fresno, California. Sus padres provienen de San Mateo Tunuchi, Oaxaca. Tiene 23 años y es estudiante de psicología en Fresno State. Lo que nos interesa presentar en este caso es la posibilidad de que una persona nacida en Estados Unidos pueda enfocarse en sus estudios antes que entrar a trabajar en el campo. Desde luego, por ser ciudadana cuenta con todos los beneficios que otorga el Estado para estudiar con ayuda económica. Sin embargo, sus padres no se desincorporaron del trabajo jornalero y Catalina se vio obligada a cuidar de su hermana pequeña mientras sus padres salían a piscar, de tal suerte que, cuando le pregunté si ella consideraba estar experimentando o haber experimentado la etapa de la juventud, contestó lo siguiente:

No por completo ... Depende, depende de cómo son los padres, pero no, por la razón porque nosotros teníamos que cuidarnos a cada uno, mi hermana mayor tenía que cuidar de los más chicos y cuando nosotros estábamos más grandes pues vino la más chica, y todos teníamos que fallar [a la escuela] y tomar la responsabilidad de cuidarla, de fallar un día de clases y cuidarla a ella, teníamos esa responsabilidad... Creo que depende, depende de la experiencia de los padres, depende de los niños, depende de la situación, y cada niño, con cada niño las cosas cambian, las cosas eran más difíciles con los primeros dos y tres, pero ya conmigo y con la chica cambiaron las cosas. No lo tuve tan fácil, no eran fácil las cosas, especialmente financieramente, pero ya con la más chica, pues ella tiene un IPhone, IPaid Nano, el más grandecito, ahm tiene un PSP un play station, un PS Two. Ha ido al cine varias veces de la edad que nosotros íbamos, ella lo tiene bueno, y le digo, le explico y le digo no te quejes, porque sí, hay veces que no puedes salir con tus amigas, pero tú has hecho más de lo que cualquiera de nosotros hemos hecho juntos, tú tienes más libertad, aunque sí entendemos que quieres estar con las amigas pero a veces no se puede. Pues eso depende, no tuvimos la juventud o la niñez como la mayoría. (Catalina [pseudónimo] 9 de septiembre de 2010, Madera, Ca.).

Caso Mónica: Por último, presento el caso de Mónica, quien es de origen mixteco. Tiene 33 años y llegó a 
los seis a vivir al Valle de San Quintín. Mónica tiene una larga experiencia en el trabajo relacionado con la agricultura de exportación. Ha transitado por diversos puestos, desde trabajar como jornalera hasta realizar procesos sofisticados en floricultura. A lo largo de su relato, Mónica nos permite observar cómo su condición de mujer resultó un obstáculo para que su padre aprobara que ella pudiera estudiar. No obstante la situación de conflicto que enfrentaba Mónica, continuó estudiando al mismo tiempo que trabajaba para apoyar económicamente a su familia y pagar los gastos escolares.

Susana: Déjame te pregunto: itú sientes que has vivido esa etapa de la juventud?

Mónica: Pues yo pienso que en su momento, como que creo que no. A la vez creo que no porque, bueno, yo he mirado que todos los jóvenes viven, no sé de qué forma viven su juventud, porque ellos en el trabajo, hasta en el trabajo tienen novios, vacilan, juegan y esto, y yo, como que después de mis 16 años, 17 años más o menos, me enfoqué como que a trabajar y a tratar de aprender, de lo que fue de los 18 años, bueno de 17 años que estaba en el INEA no quise tener mucho como digamos, no quise acercarme mucho con los jóvenes, socializar mucho con ellos porque no quería tener más problemas en mi casa

Susana: ¿O sea ya tener un novio ya hubiera sido un problema?

Mónica: Sí, sí. Creo que sí, y como que salir y eso, yo no quería tener ningún problema. (Mónica [pseudónimo] 17 de agosto de 2010, San Quintín, B.C.).

A partir de estos casos, hipotéticamente podríamos decir que la etapa de la juventud, como parte del ciclo de vida de los jóvenes mixtecos y zapotecos, está cruzada por circunstancias que devienen de la familia y el trabajo, las cuales condicionan la posibilidad de experimentar esta etapa de la vida.
En primer lugar, la familia se presenta como un actor que vigila y regula tanto a hombres como a mujeres, pero de manera diferenciada. A las mujeres se les advierte de que no deben tener hijos a temprana edad y mucho menos novios, esto con la idea de que "no echen a perder su vida". La prohibición del noviazgo y de la posibilidad de salir con amigos es una situación recurrente para el caso de las mujeres. En los hombres la regulación es menor, sin embargo, muchos de ellos se ven obligados a dedicarse a trabajar en el campo como parte del apoyo económico familiar y de sus funciones masculinas. Tal situación afecta de igual manera la posibilidad de experimentar la etapa juvenil.

En segundo lugar, la dinámica del trabajo jornalero se presenta como una gran condicionante para que los hijos logren afianzar la etapa de la juventud tal y como la conocemos en otros sectores de la población, donde existen ciertas libertades y autonomía para realizar actividades en conjunto con otros jóvenes, como tener amigos, salir, estudiar, etcétera. En los relatos de los jóvenes es recurrente observar que, como ellos se dedicaron a trabajar, no tuvieron tiempo para disfrutar con otros compañeros de su edad excepto en algunas ocasiones en el campo con sus pares que también trabajaban en el campo, durante sus labores en la misma cuadrilla o a la hora del "lonche". En otros casos, puede observarse que muchos de ellos, por la falta de ayuda de sus padres para poder estudiar, se veían obligados a combinar la actividad laboral con la escolar, utilizando su cheque para los gastos de uniforme, útiles y cuotas escolares. En muchas ocasiones, asistir a la escuela representaba un grave problema frente a la familia, particularmente si quien decidía estudiar era una mujer, debido a que existe la idea de que "la escuela no es para nosotros" y menos para una mujer.

Finalmente están los casos de aquellos jóvenes que no trabajaron. Este grupo corresponde a los que ya nacieron en el lugar de destino en ambos valles. Los jóvenes de esta segunda generación de migrantes tuvieron más facilidades para incorporarse a la escuela, sin embargo, 
debían dedicarse al cuidado de sus hermanos pequeños o a algunas actividades del hogar hasta el momento en que sus madres o padres regresaban del campo, pues las jornadas laborales eran tan extensas que el cuidado de los más pequeños muchas veces se encomendaba a los hermanos más grandes, si es que éstos no habían entrado a trabajar al campo.

\section{Conclusión}

En este trabajo se trató de exponer una idea general sobre lo que podríamos llamar la genealogía del trabajo jornalero a partir de las memorias de una generación de jóvenes mixtecos y zapotecos que nacieron o crecieron en espacios de migración. Un tema que resultó de nuestro interés fue indagar la forma en que estos jóvenes concebían la etapa de vida llamada juventud. En este sentido, fue importante observar que, para algunos jóvenes, esta etapa cercana al rango de edad entre los 16 y los 18 años, no ha sido experimentada con la "plenitud" que ellos imaginan como el ideal de "ser" joven.

Este ideal casi siempre está asociado a no tener compromisos familiares ni laborales y a estarúnicamente enfocados en los asuntos de la escuela, salir con los amigos y tener mayor libertad. Para los dos tipos de generación que presentamos (segunda generación e intermedia), los procesos de migración y de inserción laboral a los campos agrícolas han tenido un efecto importante en el desarrollo de sus etapas de vida, aunque, como vimos, las experiencias se diferencian de acuerdo al estatus migratorio. En el caso de la migración interna, una divergencia fundamental entre los que llegaron y los que nacieron en el Valle es el haber vivido en campamentos o en colonias. En este caso, el tipo de residencia dio lugar a la creación de experiencias de incorporación diferenciadas. Para el caso de la migración internacional, el estatus legal migratorio imprime características y experiencias de diferente tipo entre los jóvenes.
Por esta situación, en un principio retomamos de manera general el tema de la generación: iqué es lo que caracteriza a esta generación de jóvenes mixtecos y zapotecos? ¿Se puede hablar de una generación como tal? ¿Hay experiencias históricas compartidas que nos permitan tratar a estos grupos de jóvenes como una generación definida? Si bien una generación no puede ser concebida de manera homogénea debido a que hay diferencias de clase, de género y étnicas, los relatos de los jóvenes nos permiten intuir que existe una "situación de generación”, como lo menciona Mannheim, dado que las memorias compartidas de procesos vividos a lo largo de su experiencia migratoria han ocurrido alrededor de las décadas de los ochenta y noventa marcando el curso de sus vidas.

Asimismo, estas situaciones de generación nos permiten observar conflictos intergeneracionales entre los jóvenes, sus padres y abuelos. Mientras estos últimos concebían la migración solamente como una fuente para obtener ingresos, los jóvenes se incorporan al espacio de una manera distinta. Es decir, crean pertenencias a partir de su interacción con la nueva sociedad receptora, e incluso son críticos con sus padres en diversos sentidos. Por ejemplo, existen diferencias entre padres e hijos en relación con la importancia de la educación. Este aspecto es de suma relevancia pues evidencia cambios tanto en las formas de concebir las relaciones de género, como en la posibilidad de incorporarse a nuevos espacios socioeconómicos. Según los relatos presentados, la educación aparece como un factor que detona diferencias intergeneracionales, donde los padres y los hijos debaten sobre "lo que debe estar haciendo un hombre o una mujer", sobre su pertenencia étnica y de clase, y sobre la imposibilidad de incorporarse a nuevos segmentos de la sociedad receptora.

Hasta aquí hemos tratado de presentar algunos hallazgos preliminares sobre lo que acontece con las nuevas generaciones de jóvenes indígenas que han crecido y nacido en los lugares de migración elegidos por 
sus padres para asentarse. Lo que se propone, por tanto, es una línea de investigación que puede ser analizada más profundamente y que se ha abordado poco en la literatura sobre jóvenes migrantes en la frontera México-Estados Unidos.

\section{Notas}

${ }^{1}$ En la actualidad son por lo menos cinco los grupos étnicos que sobresalen en estos circuitos migratorios en ambos lados de la frontera: mixteco, zapoteco, triqui, náhuatl y chatino (Estudio de Trabajadores Agrícolas Indígenas, ETAI).

${ }^{2}$ El número de inmigrantes en Estados Unidos entre 1981 y 1990 ( in considerar la legalización de IRCA) ha sido el mayor de los últimos 60 años, ya que desde la década de 1910 no se registraba una cifra igual (Verduzco, 1997).

3 El aumento de migración proveniente de diversos países, particularmente de México, llevó a Estados Unidos a tomar varias medidas de política migratoria para lograr más adelante la aprobación de una nueva Ley de Reforma de la Inmigración, mejor conocida como IRCA (Immigration Reform and Control Act), cuyo objetivo principal era la legalización de la población extranjera que se encontraba indocumentada hasta el año 1982.

${ }^{4}$ Es decir, de ser migrantes itinerantes y vivir en la propiedad del dueño de los campos, pasaban a ser residentes del Valle de San Quintín con un terreno propio.

${ }^{5}$ A partir de los últimos años, los jóvenes del Valle de San Quintín han considerado el municipio de Ensenada, ubicado en el estado de Baja California, como un centro de atracción para realizar estudios profesionales. Esto ha sido así, principalmente por la escasez de carreras ofrecidas en la Universidad Autónoma de Baja California con campus en San Quintín. En ésta, las carreras casi siempre están enfocadas a la actividad agrícola y turística. En cambio, en Ensenada hay otras opciones como comunicación o artes plásticas.

\section{Bibliografía}

Benhabib, Seyla (2002), The claims of culture. Equality and diversity in the global era, Princeton: University Press, Princeton y Oxford.

Coubès, Marie Laure, Laura Velasco y Christian Zlolniski (2009), "Asentamiento residencial y movilidad en el Valle de San Quintín: Reflexión metodológica sobre una investigación interdisciplinaria", en Rivera Sánchez, Liliana y Ascencio Fernando Lozano (coords.), Encuentros disciplinarios y debates metodológicos: la práctica de la investigación sobre migraciones y movilidades, México: CRIM/UNAM/Miguel Ángel Porrúa.

Estudio de Trabajadores Agrícolas Indígenas (ETAI) (2007-2009) y Programa Indígena de la Asistencia Rural Legal de California (CRLA, por sus siglas en inglés), 〈http://indigenousfarmworkers.org/es/ about_ifs.shtml> [10 de octubre de 2011].

Feixa, C. (1999), De jóvenes, bandas y tribus, Barcelona: Ariel. Guiardo, F. (2004), "Generaciones y juventud: una relectura desde Mannheim y Ortega Gasset", en Revista Última Década, núm. 20, pp. 11-46.

National Agricultural Workers Survey, NAWS (1991-2008), 〈http:/www.doleta.gov/agworker/naws.cfm〉 [10 de octubre de 2011].

Portes, A. y Rubén, R. (1996), Immigrant America: A Portrait, Berkeley, California: University of California Press.

Verduzco, Gustavo (1997), "La migración mexicana a Estados Unidos: estructuración de una selectividad histórica", en Tuirán, R. (coord.), Migración MéxicoEstados Unidos: continuidad y cambio, México: CONAPO, pp. 11-32.

Velasco, L. (2005), Desde que tengo memoria. Narrativas de identidad en indígenas migrantes, México: El Colegio de la Frontera Norte/Fondo Nacional para la Cultura y las Artes.

Velasco, L. (2004), "Identidad y migración. Relato de vida", en Revista Historia, Antropología y Fuentes Orales, núm. 31, pp. 75-98. 
Velasco Ortiz, Laura (2002), El regreso de la comunidad: migración indígena y agentes étnicos, los mixtecos en la frontera México-Estados Unidos, México: El Colegio de México/El Colegio de la Frontera Norte.
Zhou, M. (1997), "Growing Up American: The Challenge Confronting Immigrant Children and Children of Immigrants", en Annual Review of Sociology, vol. 23, pp. 63-95. 